\title{
In situ Insights into the Uncorking and Oxidative Decomposition Dynamics of Gold Nanoparticle Corked Carbon Nanotube Cups for Drug Delivery
}

\author{
Stephen D. House ${ }^{1}$, Christopher M. Andolina ${ }^{1}$, Seth C. Burkert ${ }^{2}$, Alexander Star ${ }^{2}$ and Judith C. Yang ${ }^{1}$ \\ 1. Department of Chemical and Petroleum Engineering, University of Pittsburgh, Pittsburgh, PA (USA). \\ 2. Department of Chemistry, University of Pittsburgh, Pittsburgh, PA (USA).
}

Nitrogen-doped carbon nanotube cups (NCNCs) are an intriguing material for drug-delivery applications due to their cup-shaped morphology and their propensity for chemical modification. NCNCs can readily uptake small molecules, which can be effectively sealed within the inner cavity through the formation of nanoparticles (NP) on the open end of the cup. The resulting nanocapsules can then undergo oxidative biodegradation through biologically available peroxidase enzymes, [1] effectively releasing their loaded cargo. This mechanism has been applied for Au NP-corked NCNCs loaded with chemotherapeutic molecules for immunotherapy in melanoma models.[2] However, a better understanding of the mechanisms of cargo release - through uncorking and/or nanotube degradation - is paramount for improving their effectiveness as drug delivery platforms. The environmental transmission electron microscope (ETEM) is a powerful tool to directly investigate these questions as it combines subnanoscale spatial resolution with the ability to image Au NP-NCNCs under similar gas, thermal, and liquid environments as their intended applications. Here we present our in situ ETEM study of the dynamic oxidation-dependent uncorking and degradation of Au NP-NCNCs.

NCNCs are synthesized using a liquid (a mixture of xylenes, acetonitrile, and ferrocene) injection chemical vapor deposition method at a temperature of $800{ }^{\circ} \mathrm{C}$ for $1 \mathrm{~h}$. The resulting stacked NCNCs are separated into short stacked segments [3] by sonication in a mixture of $\mathrm{H}_{2} \mathrm{SO}_{4}$ and $\mathrm{HNO}_{3}$ for $4 \mathrm{~h}$ followed by probe tip ultrasonication for $1 \mathrm{~h}$.[4] The individual and short stacked NCNCs are corked with AuNPs through a sodium citrate reduction of hydrogen tetrachloroauric acid at $75^{\circ} \mathrm{C}$ for $2 \mathrm{~h}$. Aqueous solutions of the NCNCs are drop-cast onto ultrathin carbon TEM grids (Ted Pella, Inc.) and MEMs (Protochips, Inc.) chips. We studied the structure of the Au NP-capped NCNCs and the dynamics of their uncorking and subsequent degradation in situ using a Hitachi H9500 ETEM equipped with a $\mathrm{LaB}_{6}$ source and operated at 200-300 kV. The microscope is equipped with a differential pumping system and a homebuilt gas injection system. Experiments were performed using a double-tilt heating holder (Hitachi High Technologies, Inc.) and a MEMs-style heating holder (Protochips, Inc.). We explored maximum temperatures and $\mathrm{O}_{2}$ pressures of $750{ }^{\circ} \mathrm{C}$ and $10^{-2} \mathrm{~Pa}$, respectively.

Structural examination of the NP-capped NCNCs revealed a multilayered tube structure, often with multiple internal compartments. The Au NP morphology consisted of either a cap (interacting only with the uppermost lip of the NCNC) or plug (extending deeper into the tube). The origin of this heterogeneity and the underlying chemical interactions between the cap/plug and the NCNCs are currently under investigation. The corked NCNCs exhibited a remarkable tolerance to heat up to temperatures of $800^{\circ} \mathrm{C}$, however, the internal structures of the carbon tubes were observed to restructure at temperatures of $500{ }^{\circ} \mathrm{C}$. The segmented cavities changed from a truncated cone shape to spherical cavities, decreasing in apparent volume by $50-60 \%$. This may be a result of increasing pressure on the walls from the encapsulated cargo as it is heated or due to thermally induced stresses. Au-NP caps were shown to be slowly "pulled" into the tubes at temperatures of $700-750{ }^{\circ} \mathrm{C}$ at rates of $\sim 0.1 \mathrm{~nm} / \mathrm{s}$. Upon exposure to $\sim 1 \mathrm{~Pa}$ of $\mathrm{O}_{2}$, however, these ingrown $\mathrm{Au}$ plugs were ejected from the tubes at a temperature- 
dependent rate, increasing 2-3 orders of magnitude from $0.02-0.03 \mathrm{~nm} / \mathrm{s}$ at $400{ }^{\circ} \mathrm{C}$ to $7-33 \mathrm{~nm} / \mathrm{s}$ at 800 ${ }^{\circ} \mathrm{C}$ (e.g., Figure 1a). This uncorking was not continuous, but rather a stepwise, punctuated nature. Importantly, the onset of uncorking occurred prior to any observed oxidative degradation of the NCNC.

Significant oxidative decomposition of the NCNCs under the listed $\mathrm{O}_{2}$ pressure required temperatures $\sim 500^{\circ} \mathrm{C}$. The exterior walls and any interior walls of cavities that were exposed (e.g., from a breach) exhibited the same manner of attack. The oxidation primarily initiated at the rims (exterior wall) and cup bottoms (interior wall) of each of the NCNCs and progressed along the cup walls (e.g., Figure 1b). Oxidation of the exterior walls typically proceeded at a slower rate and after a period of delay, attributed to the presence of an amorphous carbon layer on the outside of the tube, which could act as a sacrificial barrier hindering oxidation. In all cases, once oxidation began, it occurred along the entire length of the tube, with no preference for the ends. In contrast, interior cavities without an obvious breach exhibited an isotropic thinning of their walls, indicating a different mechanism was involved. This behavior is currently under investigation. It is important to note that the oxidative degradation of the NCNCs in aqueous solution may not necessarily proceed by the same mechanisms as these solid-gas reactions. For this reason, ETEM experiments are underway using an in situ liquid cell holder (Hitachi) to study the uncorking and degradation reactions in the enzymatic solution environments. Surface chemistry changes are being explored by in situ X-ray photoelectron spectroscopy experiments to correlate with the gasbased ETEM structural changes. The knowledge gained from these studies will enhance the tailoring of the Au NP-corked NCNCs properties for a more effective therapeutic delivery.[5]

References:

[1] I Vlasova, et al., Toxicology Applied Pharmacology 299 (2016), p. 58.

[2] Y Zhao, et al., Journal of the American Chemical Society 137 (2015), p. 675.

[3] Y Tang, et al., Journal of Physical Chemistry C 117 (2013), p. 25213.

[4] Y Zhao, et al., ACS Nano 6 (2012), p. 6912.

[5] This work was supported by NSF DMR grants \#1508417 and 1410055, NSF DMREF \#CHE1534630, NSF career award \#0954345, NIH R01ES019304, and the ETEM Catalysis Consortium (ECC, funded through U. Pitt and Hitachi High Technologies). The microscopy work was performed at the Peterson Institute for NanoScience and Engineering (PINSE) Nanoscale Fabrication and Characterization Facility (NFCF) at U. Pitt. The authors would like to acknowledge Mr. Matt France and Dr. Susheng Tan for their assistance with the experimental setup.

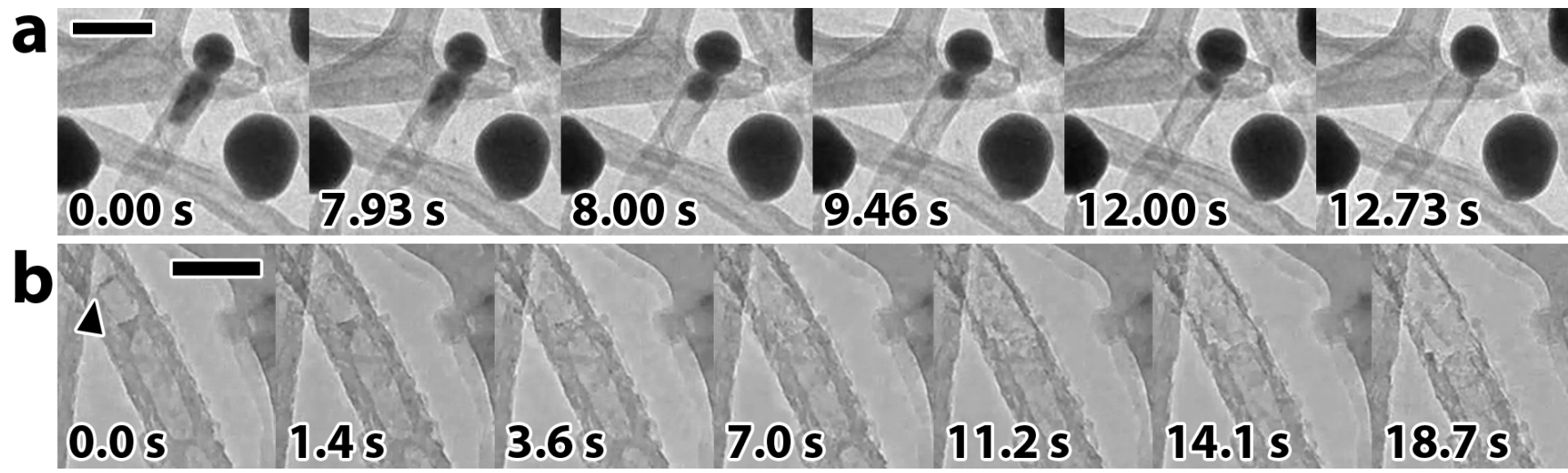

Figure 1. Selected stills from in situ ETEM videos showing examples of (a) uncorking of an Au NP plug $($ scalebar $=100 \mathrm{~nm})$ and $(b)$ oxidation of an NCNC (scalebar $=50 \mathrm{~nm})$. The triangle indicates the bottom of an exposed stacked nano-cup, one of the initiation sites. 